\title{
Cicero sententiam rogatus tandem ad Relationes Redit (CiC. PHiL. 7.27)
}

\author{
F. X. Ryan
}

\begin{abstract}
The very last sentence in Cicero's Seventh Philippic has been faultily transmitted, probably not miswritten through mere inattention, but misread by a copyist who was expecting something else, or even miscorrected by a proofreader or reader unmindful of the opening of the speech.
\end{abstract}

M. Tullius Cicero medio fere mense Ianuario post annos viginti quam consul fuerat orationem in senatu habuit res ad patres relatas egredientem, quam ob causam non prius consedit quam alienae sententiae adsensus est: quibus de rebus refers, $P$. Servilio adsentior (§ 27). ${ }^{1}$ Sed tenebant senatum tunc duo homines, consul C. Pansa $(\S \S 5,12,27)$ et tribunus plebis nobis ignotus, qui varie rettulerant: de Appia via et de Moneta consul, de Lupercis tribunus plebis refert $(\S 1) .{ }^{2}$ Nullo igitur modo potuit orator perorans dicere refers, quod scribendum est refer $<t i>s .^{3}$ Paululo ante consulem adloqui coeperat ( $\$ 27$ : te ipsum, Pansa, moneo) adloquebaturque eum solum donec animum ad res relatas attendit. Itaque

${ }^{1}$ Vide editionem e D. R. Shackleton Bailey († MMV) novissime recognitam, curantibus J. T. Ramsey et G. Manuwald: Cicero, Philippics 7-14 (Harvard University Press: Cambridge, Mass. 2009); dissensio de his verbis inter doctos, quoad scimus, nullo tempore orta est. Ut apparatus criticus apud Paulum Fedeli, In M. Antonium Orationes Philippicae XIV (Teubner: Leipzig 1982), ostendit, aliquot codices imperfectas atque peiores lectiones continent: quibus...adsentior om. nsv de rebus om. bt I adsentior om. $\mathbf{t}$

${ }^{2}$ Willems (1885: 133-34 et 134 n. 1) haec verba proferens ac existimans ea ad "l'ordre du jour" pertinere credidit nihilo minus gerentes diversos magistratus ordine ("à leur tour") rettulisse. Quam repugnantia iudicia! Ex hac doctrina locus adlatus duo vitia exhibit. Oportuit Belgicum doctum et post consul verbum refert supplere et post tribunus plebis verbum refert traditum in referet mutare: de Appia via et de Moneta consul $<$ refert $>$, de Lupercis tribunus plebis refer<e $>t$. Sed plane consularis parvo intervallo post relationes ab consule factas rogatus omnino affirmare non potuit tribunum plebis seriore diei hora relaturum esse; brevis autem ille senatus hibernus fuit, quia nox consilium diremit (cf. Varr. ap. Gell. 14.7.8 et de Reatini testimonio v. Ryan [2002]). Pelham (1891: 628) verba exordii melius interpretans iudicavit: "It would seem from Cic. Phil. vii.1, that if the questions were small ones, the references of both consuls and tribunes might be put conjointly to the house." Ex hoc loco uno argumentum totum credibile elicuit, sed exstat in Latinis fontibus exemplum talis consultationis alterum: mense Ianuario anno DCLXXXXVIII consules tribunusque plebis (Cic. Fam. 1.2.2) senatum de rege Alexandrino consuluerunt (cf. Cic. Q.fr. 2.2.3). Mommsen (1888: 954-55 et 955 n. 1), qui alio loco (919 n. 6) eventa in senatu per hoc triduum confuse enumeravit, septimam Philippicam initio omittens et his litteris praecipue fretus declaravit: "es haben aber auch ungleichartige Beamte Verhandlungsgegenstände in der Weise zur Umfrage gebracht, dass bei den Voten auf alle zugleich eingegangen werden musste." Cum res illo die ad senatum relata haud parva fuerit, consul tribunusque plebis contra Pelhamensem opinionem numquam prohibiti sunt communiter referre. Mommsen (p. 955 n. 3) mox commemorans septimam Philippicam demonstrantem "dass minder wichtige Vorlagen oft zusammengefasst wurden" aperuit quid de earum relationum coniunctione sentiret: "Ciceros siebente philippische Rede...beweist zugleich, dass gleichzeitig ein Consul Strassen- und Münzangelegenheiten, ein Volkstribun eine die Luperker betreffende (sc. Vorlage) zur Verhandlung stellen konnte."

${ }^{3}$ Unus duorum arbitrorum indicavit: "this sentence has not caused problems before." At aliqui quibus de rebus refers magis sensum e sensu quam e verbo verbum transferentes (W. C. A. Ker, Loeb ed., Cambridge, Mass. 1926: "On the motion submitted"; D. R. Shackleton Bailey, Cicero, Philippics, Univ. of No. Carolina, Chapel Hill, 1986: “On the business before us") mendum sponte aut non sponte tegunt; Willems (1885: 134 n. 1) numerum verbi refers singularem percipiens ("ces mots-ci adressés au consul Pansa") non tamen consideravit num id recte traditum esset.

STUDIA HUMANIORA TARTUENSIA vol. 11.A.4 (2010)

ISSN 1406-6203 · http://www.ut.ee/klassik/sht/ 
haec est errati origo, non neglegentia in scribendo, sed in legendo, nisi verbum refertis recte scriptum ac lectum, sed pro falso habitum, infeliciter emendatum est.

Hic erat finis nostrae quaestionis. Arbitris ab curatoribus horum studiorum selectis emendationem temptatam aegre patientibus, ${ }^{4}$ nunc quaerimus num praetermissio tribuni plebis in fine orationis aliter tractanda sit. Alias correctiones divulgamus duas, quae verbum refers non violarent. Fieri potest ut culpa in exordio insit. Si mentio tribuniciae relationis interposita esset, eam secluderemus: de Appia via et de Moneta consul [de Lupercis tribunus plebis] refert. At constat codicem V (= cod. tabularii Basilicae Vaticanae H. 25, saec. IX) istiusmodi in verba tradita inserta non habere. ${ }^{5}$ Praeterea verba de quibus nunc dubitamus speciem scholii in margine scripti non habent, illa enim glossemata voces ac res vix intellegibiles quam brevissime exponentes nihil amplius praebent. Potest etiam fieri ut culpa in peroratione insit, verbo refers excepto. Dissimilis quidem modus referendi in senatu tredecim annis ante fuerat, nam consules Marcellinus Philippusque (Cic. Fam. 1.2.2) ${ }^{6}$ et tribunus plebis Lupus omnes de causa regia (cf. Cic. Fam. 1.5a.2) rettulerunt, hic exactius de Pompeio (Cic. Fam. 1.2.2, cf. 1.1.3), id est, varie de eadem re. Ergo Cicero, qui tum Q. Hortensio adsensus est, sententiam rogatus necessarie omnes magistratus eodem tempore adloquens dixit: "qua de re refertis, Q. Hortensio adsentior." Anno urbis conditae DCCXI contra ea consul tribunusque plebis non solum varie, sed etiam de variis rebus rettulerunt. Tres relationes, quarum duae ab consuleideo Cicero de iterum pro Moneta posuit-factae sunt; ${ }^{7}$ tribus sententiis dictis quisque sententiam rogatus potuit ter adsentiri. ${ }^{8}$ Certo Cicero Pansam solum verbis quibus de rebus adfari potuisset, consulares enim relationes, ut modo diximus, duae numero erant. ${ }^{9}$ Si Cicero consulem intuens P. Servilio adsensus esset, statim aspiciens ad tribunum plebis de eius relatione dixisset. Q. quidem Fufius Calenus illo die in senatum venisse videtur $(\S 5)$. Cuius sententiam de Lupercis Cicero sequi fortasse maluisset. ${ }^{10}$ Eo aliquis dicat adsentationem M. Tullii amplificandam esse ac post verbum adsentior ultimum in oratione traditum ponat haec verba: <qua de re refers, Q. Fufio adsentior. $>$ Verum haec curatio paene gravior quam morbus videtur, quia verba suppleta nusquam confirmantur. Et nihil favet longo supplemento praeter singularem verbi refers traditi numerum. At aliud ac simplicius remedium repertum est.

\footnotetext{
${ }^{4}$ Unus arbiter scripsit: "above all, evidence has to be adduced that a consul and a tribune of the plebs are addressed together in the plural like this elsewhere." Cui rei etiam alter arbiter consensit: "It won't do to emend...simply on the basis of Phil. $7 \S \S 1$ and 27." Hic arbiter inter cetera desideravit aliam sententiam in senatu dictam "that embraces 2 such disparate relationes as the via Appia and Mint, on the one hand, and the Luperci, on the other." Consulis una cum tribuno plebis referentis exemplum anni DCCIIII tertium ultimumque memoriae produnt Graece scribentes eruditi posterioris aetatis (App. BC 2.30, cf. Plut. Pomp. 58). De his omnibus consultationibus alia oratio nulla restat. Optimus autem huius orationis codex Shackleton Bailey (1986: xiv) iudicante "is the work of an ignorant scribe."

${ }^{5}$ Cf. Shackleton Bailey (1986: xiii-xiv): " $V$ is an 'honest' manuscript, clear of interpolation, to which $D$ [= consensus codicum bcnstv, id est, ceteri codices, Cusani quaedam excerpta continente codice deterioribusque exceptis] has been heavily subjected."

${ }^{6}$ Mommsen (1888: 955 n. 1) mentionem Marcellini soli fecit.

${ }^{7}$ Aliter Willems (1885: 134 n. 1), qui consuli "la première relatio" attribuit; etiam Mommsen (1888: 955 n. 3)

"Combination disparater Gegenstände durch denselben Vorlegenden" una sola "Relation" contineri sivit.

${ }^{8}$ De Ciceronis in dicendo loco, videlicet quarto vel quinto vel sexto, C. Pansa et A. Hirtio consulibus, v. Ryan (1998: 273 et nn. 192-193).

${ }^{9}$ Cf. King (1868: 191), qui autem duplicem relationum consularium numerum non intellexit, de verbis quibus de rebus: "the Appian way, and the Mint, the subjects of the Consul's motion."

${ }^{10}$ De gratia Caleni in senatu primi rogati v. Ryan (1998: 328-40).
} 
Propterea legamus correctionem primo propositam, quoniam mendum facile explicatum tollit per additas duas tantum litteras: quibus de rebus refer $<t i>s$, P. Servilio adsentior.

Franciscus X. Ryan

Los Angeles, California

E-mail:fxryan@gmx.de

\section{Scripta Posteriorum}

King, J. R. (1868) The Philippic Orations of M. Tullius Cicero. Oxford: Clarendon Press.

Mommsen, T. (1888) Römisches Staatsrecht, Band III.2: Bürgerschaft und Senat (Schluss). Leipzig: Hirzel.

Pelham, H. F. (1891) 'Senatus.' - Smith, W.; Wayte, W.; Marindin, G. E. (edd.), A Dictionary of Greek and Roman Antiquities, 3rd ed. London: Murray, 2.620-36.

Ryan, F. X. (1998) Rank and Participation in the Republican Senate. Stuttgart: Steiner.

Ryan, F. X. (2002) 'Varro bei Varro? Der Brief an Oppianus und das Handbuch für Pompeius.' - Hommages à Carl Deroux II: Prose et linguistique, médicine. Bruxelles: Coll. Latomus, 372-385.

Willems, P. (1885) Le Sénat de la république romaine, tome II: Les Attributions du Sénat, $2^{\text {ème }}$ ed. Paris: Thorin. 